\title{
Les dimensions des crises : Analyse de deux études de cas sous les approches processuelle et événementielle
}

\author{
Aline P. Pündrich* \\ Doctorante en Sciences de Gestion - Allocataire/Monitrice \\ Centre de Recherche Magellan - IAE - Université Jean Moulin Lyon 3 \\ 6 Cours Albert Thomas - 69008 - Lyon, France \\ Tel. : + 330686645893 \\ E-mail : apundrich@hotmail.com \\ Olivier Brunel \\ Maître de Conférences \\ Equipe de recherche IRIS - IAE - Université Jean Moulin Lyon 3 \\ Téléphone : 0478787642 \\ E-mail : brunel@univ-lyon3.fr \\ Luciano Barin-Cruz \\ Professeur Adjoint \\ HEC Montréal - Canada \\ 3000, Chemin de la cote-Sainte-Catherine \\ Montréal, QC H3T 2A7, Canada \\ E-mail : $\underline{\text { lbarincruz@uol.com.br }}$
}

* Pour toute correspondance

Résumé : En raison de leur caractère protéiforme, les cas de crise sont uniques et ils exigent des actions spécifiques. Afin d'offrir aux gestionnaires un cadre de référence pour mieux les affronter, de nombreux auteurs ont tenté d'identifier et de classifier les crises. Ainsi, deux approches principales peuvent être repérées : celle qualifiée d'événementielle (plutôt centrée sur les éléments déclencheurs des crises et ses conséquences) et celle caractérisée de processuelle (qui situe la crise dans un contexte plein de significations, acteurs et victimes). L'objectif de ce travail sera d'analyser une crise organisationnelle dans deux entreprises (française et brésilienne) en faisant cohabiter ces deux approches. Les résultats indiquent, parmi d'autres aspects, que l'approche processuelle permet une visualisation beaucoup plus complète des situations critiques par rapport à l'approche événementielle. En plus, l'analyse des dimensions de crise montre que une approche systémique des événements de crise, la capitalisation sur les expériences vécues et une posture optimiste et proactive des gestionnaires ressortent de cette étude comme éléments clé de la gestion des crises. 


\section{INTRODUCTION}

Les événements de crise se caractérisent par des circonstances imprévisibles; des moments de perturbation et d'incertitude qui peuvent agir comme un révélateur d'éléments latents dans la vie quotidienne des organisations ou encore comme un «effecteur » exogène, dû à son pouvoir de transformation (Morin, 1976 ; 1994). Ces dernières années, les études concernant les crises ont pris des chemins différents selon leurs définitions (Hermann, 1963; Mitroff et all., 1987 ; Forgues, 1996 ; Pearson et Clair, 1998), ce qui a aussi influencé la façon de les analyser.

Par leur caractère multiforme, les crises concernent plusieurs domaines (Libaert, 2005) et, pour cette raison, un cas de crise est une situation unique à laquelle correspondent des actions spécifiques (Pauchant et Douville, 1994). Pour faciliter leur mise en œuvre, la littérature présente certaines classifications des crises en fonction de ses éléments déclencheurs (Mitroff et all., 1987 ; Westphalen, 1992 ; Roux-Dufort, 2003) de son caractère interne ou externe (Lagadec, 1993); ou encore selon ses « cibles » : les aspects produits, industriel et institutionnel (Ogrizek et Guillery, 1997). L'identification et la classification des crises visent à offrir aux gestionnaires un cadre de référence pour leur jugement (Lagadec, 1995) de ce phénomène incertain et évolutif (Perrow, 1984 ; Thiétart et Forgues, 1997).

Certains éléments, comme l'intensification de l'information, la prolifération technologique et la compression des temps de décision et d'action (Roux-Dufort, 2003) rendent la gestion de crise encore plus difficile. Parmi les façons d'analyser et d'affronter les crises (Allison et Zelikow, 1999 ; par exemple), la littérature offre deux approches : celle plutôt événementielle (Hermann, 1963) et celle plus processuelle (Forgues, 1996), sur lesquelles cet article se fondera.

L'objectif de ce travail sera donc d'analyser deux cas de crise organisationnelle selon les approches événementielle et processuelle, afin d'illustrer ces deux modèles théoriques (Siggelkow, 2007) et essayer de renforcer certains de leurs aspects à partir des informations collectées. Les différences existantes entre ces deux cas de crise seront également comparées. Ainsi, dans un premier temps, une rapide revue de la littérature concernant la crise sera proposée. Puis, les aspects méthodologiques de collecte et de traitement des données seront détaillés. La présentation et l'analyse des cas, ainsi que les résultats de ce travail, la discussion et les considérations finales seront enfin présentés.

\section{LES CRISES ET LEUR GESTION}


Il existe une certaine difficulté à trouver une définition de crise (Morin, 1976 ; RouxDufort, 2000) en raison du grand nombre d'éléments qui caractérisent un tel concept (Bowonder et Linstone, 1987 ; Pearson et Clair, 1998). Parce qu'il s'agit d'une notion fortement liée à la perception (Dutton, 1986) qui peuvent en avoir les acteurs concernés, une même situation peut être perçue (et définie) de différentes manières.

Certains auteurs ont une approche plutôt négative concernant la crise, l'analysant comme une occasion nocive à l'entreprise, pleine d'incidents, de surprise et de panique (Lehu, 1998 ; Bland, 1998 ; Westphalen, 1998). D’autres optent pour une approche qui prend en compte la caractéristique déstabilisatrice et ambiguë des crises et son fort climat d'incertitude, ce qui peut créer des problèmes pour la mise en œuvre des actions (Turner, 1976). Pour eux, les crises sont des situations où les causes et les effets sont inconnus (Dutton, 1986 ; Lagadec, 2000). Dans une vision plus optimiste, la crise peut être définie comme une opportunité avec un fort pouvoir d'évolution de l'organisation, un déclencheur de changements, un processus de transformation (Bryson, 1981 ; Shrivastava et all., 1988 ; Shrivastava, 1993 ; Ogrizek et Guillery, 1997 ; RouxDufort, 2000 ; Libaert, 2005).

Libaert (2005) considère la crise comme la phase ultime d'une suite de dysfonctionnements, dont les problèmes sont parfois si enracinés qu'il faut du temps pour les percevoir. Turner (1976), par exemple, présente une séquence d'événements qui, une fois associés avec un échec de prévoyance (failure of foresight) par l'organisation, peuvent résulter dans des événements critiques. Selon l'auteur, certaines catastrophes sont potentiellement prévisibles et évitables, au même temps que inattendues et turbulentes, capables de causer une crise. La littérature propose l'identification des causes des crises : les plaintes des clients, la qualité du personnel, les rumeurs, les changements rapides (Bland, 1998); et les erreurs technique/économique et sociale/organisationnel aux niveaux interne et externe des organisations (Mitroff et all., 1987 ; Shrivastava et Mitroff, 1987). Les problèmes de gestion et les dirigeants mal préparés sont souvent les causes des crises (Daigne, 1991; Roux-Dufort, 2003). Lorsqu'elles sont « installées », les crises peuvent interrompre et/ou rendre compliquée l'interaction entre les individus et les opérations présentes ou futures de l'organisation (Thiétart et Forgues, 1997). Cette situation évolue progressivement, par phases (Mitroff et all., 1987 ; Roux-Dufort, 2003).

Face à de telles particularités, la gestion des crises doit s'adapter et faire l'objet du développement d'un ensemble de méthodes permettant de voir augmenter les chances de 
maîtriser telles situations (Mitroff et all., 1987 ; Shrivastava et Mitroff, 1987 ; Lehu, 1998 ; Roux-Dufort, 2003 ; Tran Thanh Tam et Pesloüan, 2004). Les étapes de la gestion de crise peuvent être résumées ainsi (Roux-Dufort, 2003) :

Figure 1 - Les étapes de la gestion de crise

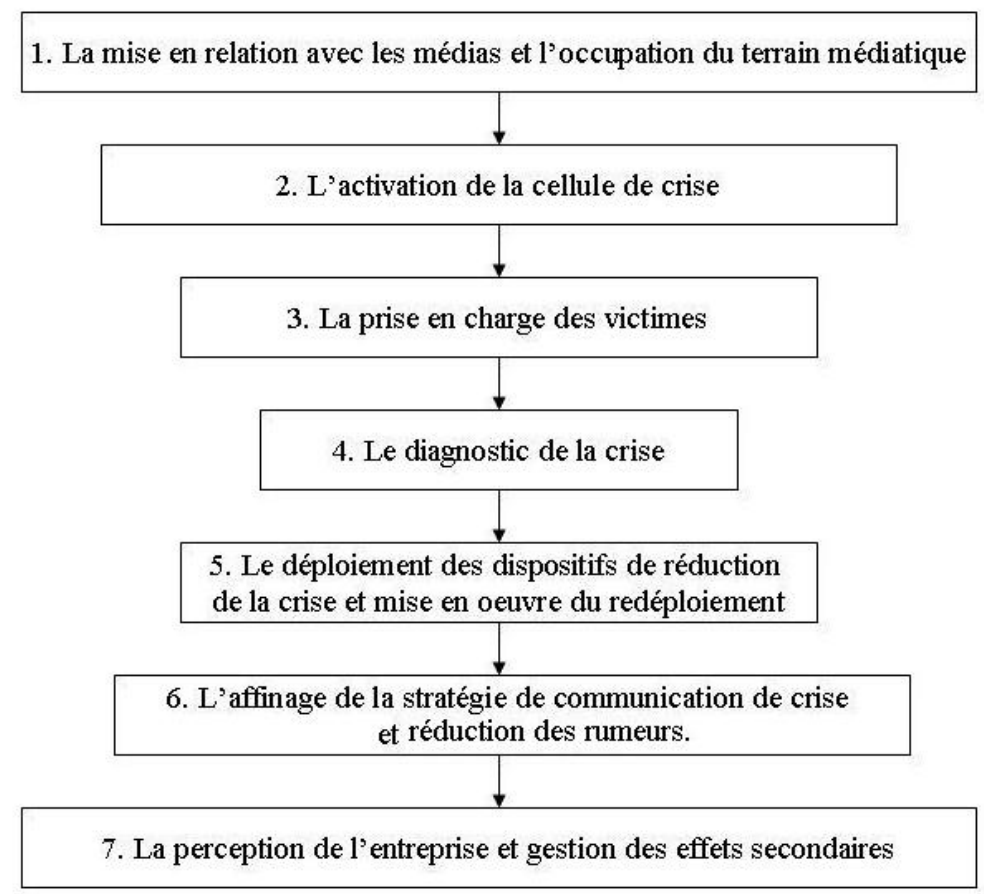

Source : Modèle construit selon les travaux de Roux-Dufort (2003)

\subsection{LES APPROCHES EVENEMENTIELLE ET PROCESSUELLE DES CRISES}

Les crises se caractérisent, entre autres, par leur effet de surprise, l'altération de la réputation de l'entreprise, la forte présence dans les medias et, souvent, par une réévaluation culturelle (Turner, 1976 ; Bowonder et Linstone, 1987 ; Pearson et Clair, 1998, Libaert, 2005). L'angoisse, l'incertitude et, souvent, l'absence de préparation peuvent également être associés à ces aspects. De façon générale, une crise présente trois caractéristiques qui la distinguent d'une situation normale de gestion, à savoir : sa condition de «processus de déstabilisation », le nombre de conséquences qu'elle peut induire et la façon par laquelle elle peut affecter le cadre référentiel de l'organisation (Roux-Dufort, 2000). Pour mieux comprendre les crises, les auteurs les ont considérées soit comme un événement (Hermann, 1963), soit comme un processus (Forgues, 1996). Lagadec (1991) illustre la différence entre événement et processus par la distinction entre accident et crise. Selon l'auteur, l'accident est un événement ponctuel, limité dans le temps et 
l'espace et qui peut faire partie d'une crise ; tandis que la crise concerne la perte du système de référence, ce qui implique des difficultés majeures en termes de contrôle et gestion de la situation. Dans ce cas, le concept d'accident est utilisé comme un événement ponctuel dont les causes et caractéristiques peuvent être déterminées dans un cadre spécifique de temps et espace. L'approche de Perrow $(1984,1994)$ n'est pas prise en compte dans ce travail, parce que l'intention n'est que d'illustrer les différences entre les approches processuelle et événementielle, ce que propose Lagadec (1991). Ainsi, dans le premier cas, l'approche événementielle se centre sur la nature de l'événement déclencheur de la crise et principalement sur ses conséquences : il y a la tendance de «privilégier les symptômes » (Roux-Dufort, 2000). Telle approche permet d'analyser la crise par ses manifestations extérieures : son principale avantage est celui d'être directement opérationnelle, de chercher à développer des moyens de réduction des conséquences de l'événement (Roux-Dufort, 2005). Ce sont des «événements rares mais inévitables » qui ne peuvent pas être une surprise, puisqu'ils font déjà partie du système de l'entreprise (Perrow, 1984 ; Forgues, 1996). Puisque l'analyse de la crise n'est possible qu'après son déclenchement, il ne reste plus aux gestionnaires qu'à adopter un comportement essentiellement réactif (RouxDufort, 2000). Lorsque la crise survient, il faut la circonscrire dans un périmètre restreint afin qu'elle perturbe le moins possible le reste de l'organisation (Forgues, 1996) et puisse être vue comme un événement isolé, ce que fournit une prise claire pour l'action (Roux-Dufort, 2005).

Figure 2 - Crise : L’approche événementielle

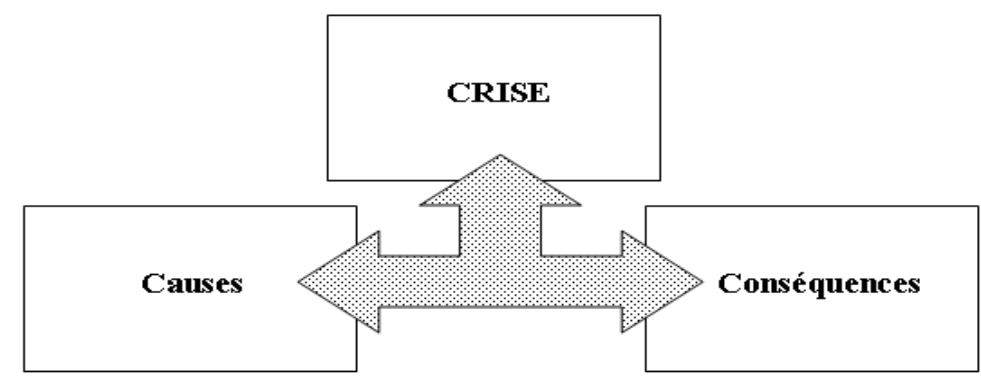

Source : Modèle construit selon les travaux de Roux-Dufort (2000)

De l'autre côté, « une approche processuelle conduit à embrasser la crise dans un laps de temps et un espace élargi » (Forgues, 1996), de façon à l'analyser comme une situation pleine de significations, d'acteurs et de victimes (Marcus et Goodman, 1991). Cette approche processuelle 
situe la crise dans un contexte plus riche et concerne ses origines, son incubation et sa «dynamique de développement » (Turner, 1976 ; Roux-Dufort, 2000). Dès lors qu'une situation critique est perçue comme le résultat de «dysfonctionnements cumulés et potentiellement repérables » dans le quotidien de l'entreprise (Thiétart et Forgues, 1997), la crise ne doit plus être considérée comme imprévisible (Bryson, 1981), puisqu'il s'agit d'un processus dont les phases d'installation, d'évolution et de développement sont, dans la plupart des cas, identifiables (Turner, 1976 ; Roux-Dufort, 2003) : la phase de déclenchement : où se retrouvent les origines de la crise, qui peuvent résulter d'erreurs des systèmes sociaux et entrepreneuriaux, d'erreurs humaines ou de la combinaison de ces éléments; la phase aiguë, qui concerne trois manifestations critiques : a) la convergence des informations et des événements vers l'entreprise; b) le dérèglement de ses routines de gestion ; c) la remise en cause de son identité, sa culture, sa mission et ses valeurs; et la phase de rééquilibrage et de changement, ou le moment où l'entreprise peut opter soit pour retourner au statu quo, soit initier un changement profond.

Figure 3 - Crise : L’approche processuelle

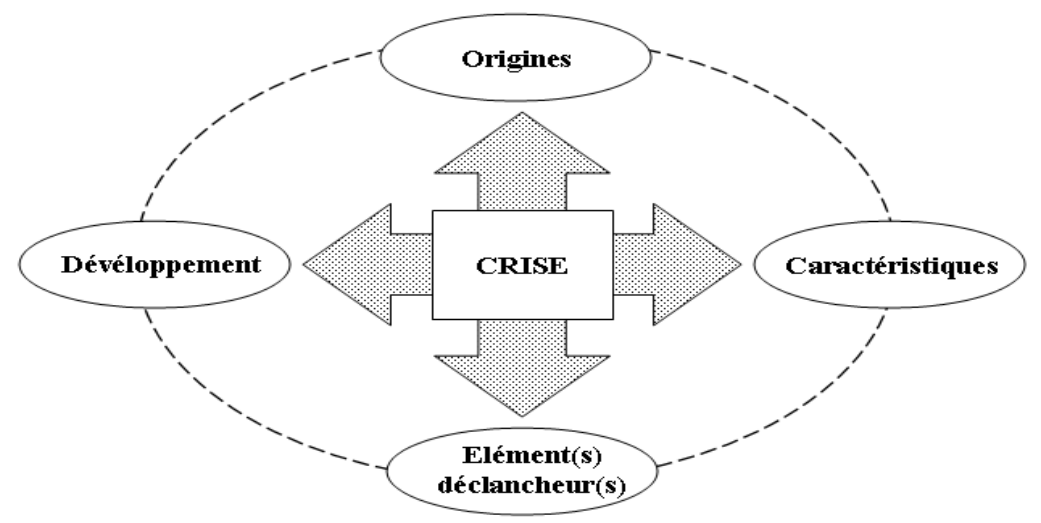

Source : Modèle construit selon les travaux de Roux-Dufort (2000)

D’après Roux-Dufort (2000), chacune des deux approches présentées (événementielle et processuelle) a des particularités qui permettent d'éclairer les situations de crise : elles peuvent même être complémentaires (Roux-Dufort, 2005).

Tableau 1 - Caractéristiques des approches événementielles et processuelles 


\begin{tabular}{|l|l|l|}
\hline $\begin{array}{l}\text { Nature de l'occurrence } \\
\text { des crises }\end{array}$ & $\begin{array}{l}\text { Surprise } \\
\text { La crise est imprévisible }\end{array}$ & $\begin{array}{l}\text { Etapes, progression dans l'intensité } \\
\text { et la visibilité } \\
\text { La crise est précédée de signes } \\
\text { avant-coureurs }\end{array}$ \\
\hline Fréquence & La crise est improbable & $\begin{array}{l}\text { Les crises sont rares mais normales } \\
\text { et inhérentes aux systèmes }\end{array}$ \\
\hline $\begin{array}{l}\text { Angles d'observation } \\
\text { des crises }\end{array}$ & Conséquences des crises & $\begin{array}{l}\text { Dynamique d'occurrence, } \\
\text { d'amplification et de résorption }\end{array}$ \\
\hline Sources des crises & $\begin{array}{l}\text { L'événement déclencheur } \\
\text { (centrée sur le symptôme) }\end{array}$ & $\begin{array}{l}\text { L'interaction non linéaire de } \\
\text { multiples facteurs et acteurs } \\
\text { (centrée sur la dynamique } \\
\text { d'occurrence) }\end{array}$ \\
\hline Explication des crises & Principe de cause à effet & Approche systémique \\
\hline $\begin{array}{l}\text { Efforts de gestion } \\
\text { des crises }\end{array}$ & Concentration sur la réaction & $\begin{array}{l}\text { Concentration sur la prévention, la } \\
\text { réaction et l'apprentissage }\end{array}$ \\
\hline $\begin{array}{l}\text { Attitude de l'organisation } \\
\text { vis-à-vis des crises }\end{array}$ & $\begin{array}{l}\text { Attentisme } \\
\text { Fatalisme }\end{array}$ & Pro activité \\
\hline
\end{tabular}

Source : Roux-Dufort (2000)

\subsection{CONSOLIDATION DES CONCEPTS}

Dans le but de réaliser les analyses auxquelles ce travail se destine, et pour donner à ce sujet sa dimension managériale, nous appréhenderons la situation de crise comme une opportunité, induisant un fort pouvoir de transformation pour l'organisation (Bryson, 1981 ; Ogrizek et Guillery, 1997 ; Roux-Dufort, 2000 ; Libaert, 2005). Les deux modèles théoriques, les approches événementielles et processuelles, seront comparés et analysés selon les modèles (Figures 2 et 3) qui ont été inspirés par les travaux de Roux-Dufort (2000), afin d'exemplifier certains éléments de ces deux approches qui servent de base pour plusieurs programmes de gestion de crise dans les organisations (Roux-Dufort, 2005). Nous utiliserons également, comme référence, les caractéristiques de chaque approche présentées par le même auteur (Tableau 1). Pour compléter ces analyses, nous utiliserons les étapes de gestion de crise (Roux-Dufort, 2003) afin de repérer la façon dont les deux entreprises ont conduit leurs crises.

\section{METHODOLOGIE}

Cette étude a été réalisée à l'aide d'une approche qualitative à partir de deux études de cas. Dans le cadre de l'analyse des crises, la recherche qualitative permet la collecte et l'utilisation de matériels empiriques qui décrivent des moments et des significations courantes et problématiques dans la vie des individus (Denzin et Lincoln, 2000). La recherche exploratoire, de son côté, permet la révision de la littérature sur le phénomène étudié selon divers niveaux et perspectives 
d'analyse. Le chercheur est basé sur l'expérience acquise dans l'interaction avec l'objet d'étude et il cherche le support théorique - souvent multilingue - dans des périodiques, des annales, des articles et des textes (Richardson, 1999). Comme stratégie de recherche, l'étude de cas contribue à la connaissance des phénomènes à des niveaux individuels, groupaux, organisationnels, sociaux et politiques. Une telle méthode permet à l'investigateur de maintenir les caractéristiques holistiques et significatives des processus réels (Yin, 2003), afin de mieux les analyser. Dans ce travail, les études de cas sont mobilisés avec l'objectif principal d'illustrer certains aspects théoriques de la gestion des crises et pouvoir ainsi les analyser de façon plus proche de la réalité des entreprises (Siggelkow, 2007).

Pour réaliser cette étude, nous avons choisi deux entreprises pétrolières, de nationalités brésilienne et française. Tel secteur d'activité a été choisi parce qu'il est souvent touché par des accidents et, comme conséquence, par des épisodes de crise. Ces entreprises sont ainsi menées à développer des actions afin de minimiser les effets de ces événements dans leur quotidien (Freitas et al., 2001). Dans ce travail, leurs contextes culturels différents peuvent permettre une analyse plus riche de la gestion de leurs crises. Ces organisations ont également une grande importance dans leurs pays, des milliers de salariés et une activité de dimension internationale. Ainsi, à cause de leur importance socio-économique, l'ampleur de leurs situations de crises et leurs structures de gestion de crises plutôt développées, ces entreprises sont l'objet de notre étude.

\subsection{COLLECTE DES DONNEES}

Ce travail a été réalisé à partir de deux types principaux de collecte de données, à savoir :

a) L'analyse bibliographique, ayant comme point de convergence les approches événementielles et processuelles des crises et avec l'objectif d'essayer de comprendre ces deux concepts pour mieux pouvoir analyser les cas de crises présentés dans ce travail ;

b) Les études de cas, réalisées sur la base de la recherche documentaire et l'analyse de matériel de presse en français, anglais et portugais, dans le but d'avoir une vision des enjeux de la gestion des crises sous différentes perspectives culturelles. Plusieurs documents ont été analysés, dont douze dossiers publiés par les entreprises concernées et par des organes gouvernementaux et syndicaux. Le matériel de presse, autour de 20 documents, a été collecté et organisé dans une perspective temporelle, pour essayer de reconstruire les événements à partir des discours des acteurs médiatiques, des journaux, des magazines et des sites d'Internet. Ainsi, l'étude s'est 
caractérisée par une recherche longitudinale a posteriori (Forgues et Vandangeon-Derumez, 2007). L'objectif étant d'identifier les crises, de les analyser en privilégiant la période de deux ans comprenant les situations de crises et de post-crise dans les deux entreprises : décembre/1999 à décembre/2001 pour l'entreprise $\mathrm{A}$ et mars/2001 à mars/2003 pour l'entreprise $\mathrm{B}$.

Les entretiens ont été réalisés pour cette étude de façon semi structurée, avec cinq cadres d'entreprises et experts en gestion de crise de nationalités brésilienne et française (dont les identités seront préservées). Le choix des interviewés s'est basé sur notre intention d'illustrer de façon pratique certains éléments théoriques de la gestion des crises depuis plus d'un angle d'observation. Ainsi, afin d'essayer de mieux comprendre le secteur pétrolière et la gestion de ses crises, nous avons eu comme interlocuteurs deux cadres qui ont participé directement aux événements de crise utilisés comme base de cette étude, les représentants des entreprises A e B (Interviewés 1 et 2); aussi bien qu'un chercheur spécialisé en gestion des crises environnementales, qui nous a apporté une conception plus académique du sujet (Interviewé 3). Un regard de la gestion des crises dans des contextes culturels différents nous a été offert par deux autres cadres, responsables pour les activités internationales de leurs entreprises. Ils nous ont présenté une vision plus pratique des crises dans les organisations (Interviewé 4 et 5).

Ces interventions ont été faites en français et en portugais, par téléphone et par support écrit, en utilisant un matériel construit à partir des références théoriques et l'analyse documentaire. Elles ont permis une triangulation (Yin, 2003) des expériences pratiques des gestionnaires concernant la gestion des crises, ce qui a permis l'enrichissement de la compréhension et de l'analyse des études de cas concernés par cette étude.

\subsection{ANALYSE DES DONNEES}

Basées sur la recherche bibliographique et documentaire, sur les entretiens et les observations faites pendant la réalisation de ce travail, les données collectées ont produit une triangulation (Yin, 2003), ce qui a permis d'améliorer à la fois la précision de la mesure et celle de la description, ainsi que d'attaquer le problème selon deux angles complémentaires (Baumard et Ibert, 2003). Tel volume de données a permis une analyse détaillée des situations de crise vécues par les entreprises choisies, ainsi que favorisé la familiarité des chercheurs avec les études de cas de façon même à faciliter la comparaison entre eux (Eisenhardt, 1989). La démarche utilisée dans le cadre de la recherche exploratoire a été basée sur la transcription et l'analyse des 
entretiens réalisés; l'analyse des données collectées selon un ordre chronologique; et l'identification, la description et l'analyse des particularités des deux cas de crise étudiés (Richardson, 1999). Nous avons opté par l'utilisation des verbatim des interviewés pour mieux illustrer certaines questions.

\section{LES ETUDES DE CAS}

Dans une première partie, certains aspects de l'activité pétrolière seront brièvement présentés, puis suivis par la description des études de cas. Celles-ci seront également exposées de façon résumée, pour que ce travail puisse se concentrer sur l'analyse des épisodes de crise selon les approches événementielle et processuelle.

\subsection{LE PETROLE ET SES RISQUES}

L'utilisation du pétrole apporte de grands risques, tant pour ceux qui travaillent avec cette substance que pour l'environnement. Depuis quelques années, les entreprises essayent de réduire les effets des accidents dans l'industrie du pétrole, soit en utilisant les avancées technologiques pour minimiser les impacts environnementaux (Paschoal, 2002), soit en cherchant plus de qualification au niveau des ressources humaines, avec le souci majeur de protéger la vie des ouvriers, spécialement dans les plateformes (Freitas et al., 2001).

Le pétrole est le produit de base le plus transporté au monde, environ $90 \%$ par voie maritime. Chaque année, près d'800 millions de tonnes de produits pétroliers sont transportés entre les ports communautaires. Entre 1992 et 1999, 593 navires ont été perdus dans le monde, dont 77 pétroliers. Il existe plusieurs causes pour ces accidents : les erreurs humaines, les mauvaises structures des navires, les conditions climatiques ou même la combinaison de ces facteurs, d'après les documents analysés pour cette étude.

\subsection{L'ENTREPRISE A ET LE NAVIRE PETROLIER NP}

Présentation : L'entreprise A est une organisation de performance internationale, présente dans plus de 130 pays, dont les activités regardent fondamentalement la production et la commercialisation de pétrole et d'électricité. Elle compte avec plus de 500 mille actionnaires et la collaboration d'environ 113 mille salariés. Cette société, à l'origine d'un projet pour la 
préservation de la biodiversité sous-marine et productrice de quelques produits biodégradables, a affronté une situation extrêmement critique concernant l'environnement.

En décembre 1999, un pétrolier a affronté une tempête et s'est cassé en deux dans une mer extrêmement forte. Au moment de l'accident, le navire se retrouvait proche d'une des côtes françaises, où ses deux parties ont coulé par environ 120 mètres de fond. Dans ses citernes, une cargaison de plusieurs milliers de tonnes de fioul lourd destinées à la production d'énergie qui ont commencé à être déversées dans les eaux quelques heures après l'évacuation de son équipage.

Dès lors, une crise a commencé pour l'entreprise A, puisqu'elle avait affrété ce navire pour transporter son produit. Les mauvaises conditions météorologiques, ajoutées à la structure du navire, sont apparues comme les principales causes de l'accident. En fait, l'état du navire n'était pas d'accord avec sa documentation et ses certifications au moment de l'accident. En se cassant en deux, ce navire a causé une marée noire de plusieurs centaines de kilomètres. Quelques jours après la tragédie, la tâche noire avait touché les côtes résultant en plusieurs milliers de tonnes de déchets.

Divers endroits de plage ont été pollués par la substance et certaines taches sont arrivées jusqu'à dix mètres de hauteur à cause des vagues. Quelques zones de sable ont été recouvertes par une couche de 5 à 30 centimètres d'huile. Paradoxalement, même les activités de nettoyage ont contribuée pour la destruction de certains éléments naturels, dû à sa grande complexité. Les coûts de cette crise ont été remarquablement hauts, non seulement en termes financiers, mais aussi pour l'image de l'entreprise, l'environnement et les individus concernés. Les travaux de dépollution et de traitement des déchets ont duré trois ans et, dans les zones les plus affectées, la commercialisation de poisson et de certains mollusques a été interdite pendant 18 mois à cause du haut niveau de pollution. En plus, plusieurs riverains et associations ont dû prendre en charge le nettoyage des côtes.

Face à la crise : Suite à l'accident, immédiatement l'entreprise A a activé une cellule de crise ainsi que déclenché des efforts pour contrôler la marée noire. Parallèlement, des enquêtes ont commencé à être menées par des parties civiles et des institutions endommagées par les événements. Concernant le discours médiatique de l'entreprise A par rapport à l'accident, son positionnement initial était de nier toute responsabilité, puisque le navire n'était pas de sa propriété. Cependant, ses parties prenantes n'étaient pas d'accord et considéraient l'organisation comme moralement responsable pour le choix du bateau et pour sa cargaison. Après quelques 
jours, beaucoup de pression de la part de l'opinion publique et quelques boycotts, l'entreprise A a accepté une partie de la responsabilité morale pour l'accident. Elle a été fortement critiquée pendant cet épisode, parce qu'elle n'avait pas donné des réponses immédiates ni reconnu promptement la gravité de la situation. Selon certains auteurs, il était déjà trop tard lorsque l'entreprise a décidé de prendre le contrôle de la situation communicationnelle face à ses publics.

Quelques mois après les événements, même 1'entreprise A avait reconnu son absence de communication et le fait qu'elle n'était pas adaptée à la situation. Ainsi, l'organisation a développé une politique bien précise pour faire face aux crises. Une unité de «pré-crise », responsable pour le monitorage des événements avec un potentiel de devenir une situation problématique, a été créée. En plus, son accident a donné origine à des nouvelles règles au sein de l'Organisation Maritime Internationale (OMI), avec l'objectif d'améliorer la sécurité du transport maritime de produits pétroliers.

\subsection{L'ENTREPRISE B ET LA PLATEFORME PETROLIERE PP}

Présentation : L'entreprise B a aussi un rôle important dans le domaine pétrolier au niveau mondial et elle se concentre sur l'exploitation des ressources de pétrole et de gaz pour la production d'énergie. Elle compte avec environ 50 mille employés, 248 mille actionnaires, des réserves d'environ 11.5 milliards de barils de pétrole et 103 plateformes d'extraction en mer. L'entreprise B est reconnue pour l'exploitation de pétrole dans les eaux très profondes et est la quinzième pétrolière au monde. Elle est également la société brésilienne qu'investit le plus dans des projets sociaux, culturels et d'éducation pour l'environnement dans son pays.

La plateforme PP était la plus grande structure de production de pétrole en haute mer au monde et elle était localisée dans une région éloignée d'une centaine de kilomètres à sud-est du littoral brésilien. Avec plusieurs étages pour des milliers de tonnes, 175 ouvriers étaient en service au moment de l'accident qui a beaucoup choqué l'opinion publique et qui a apporté des grands problèmes.

En mars 2001, la plateforme PP a subi une explosion dans une de ses colonnes de sustentation sous-marine. Cela a été suivi par deux autres explosions encore plus puissantes dans la partie émergée de la structure, ce qui a provoqué le décès de plusieurs ouvriers membres de la brigade de sauvetage. Cinq jours plus tard, la plateforme était totalement immergée à cause des explosions à l'intérieur de sa sustentation. La perte de la structure a été totale. 
L'analyse des événements nous apprend qu'un facteur crucial concernant cet accident a été l'utilisation d'un modèle de gestion par l'entreprise B qui a donné la priorité à la vitesse et aux buts de production, au lieu de privilégier les normes de sécurité du travail et d'entraînement du personnel. Des problèmes structuraux et une séquence d'erreurs humains ont été les responsables pour cet événement. Selon la presse, l'entreprise B aurait même ignoré certaines alertes de dysfonctionnement dans sa plateforme. De plus, des problèmes structuraux n'ont pas permis aux ouvriers de la brigade de sauvetage de faire leur travail en sécurité.

Face à la crise : Une des premières actions de l'entreprise B après l'accident a concerné les familles de ses collaborateurs. Avant de parler à la presse, ses responsables de gestion de crise ont contacté toutes les familles des victimes et des ouvriers présents à la plateforme au moment de l'accident afin de les donner des informations sûres et éviter la panique. Parallèlement, l'entreprise B a mobilisait plusieurs bateaux et nombreux techniciens brésiliens et étrangers afin d'essayer de contrôler la situation dans le lieu des explosions. Elle a également mené plusieurs actions pour éviter des dommages environnementales par les fuites d'huile. Comme résultat, les eaux autour de la plateforme ont été très peu contaminées.

$\mathrm{Au}$ moment de l'accident, une équipe de télévision faisait un reportage près de la plateforme PP. Ainsi, le lendemain, dès le début de la matinée, les images de la plateforme en train de plonger ont permis à la population d'accompagner en directe les difficultés des équipes de sauvetage et l'angoisse des ouvriers. Les cinq jours pendant lesquels la plateforme PP a affronté ses moments les plus dramatiques, l'entreprise B a constamment divulgué l'évolution de ses actions face à la crise. Sa cellule de crise n'a pas laissé d'espace pour des informations fausses ou des rumeurs. Telle attitude a été de grande importance, car elle a permis à l'entreprise B de préserver et renforcer ce qu'elle symbolise devant l'opinion publique nationale.

Cependant, des moments critiques ont également eu lieu, comme lorsqu'un des directeurs de l'entreprise $\mathrm{B}$ a déclaré à la presse que l'entreprise était déjà sortie de la crise. Cette déclaration été très critiquée parce que le cadre semblait d'essayer de minimiser la gravité de la situation. D'autres points négatifs ont également posé des problèmes, comme lorsque l'administration de l'entreprise a empêché la participation des représentants des ouvriers du pétrole dans la commission chargée des investigations. En plus, l'entreprise B a refusé de présenter certains documents et de donner des témoignages sollicités par les enquêteurs. 
Suite à l'accident, l'entreprise $\mathrm{B}$ a commencé un intense projet de communication afin de divulguer les résultats des investigations et les discuter ouvertement avec ses parties prenantes. Le développement de nouvelles normes de sécurité et les investissements en entraînement et en infrastructure ont aussi fait parti des actions pour minimiser les effets de cet épisode. En plus, la société a augmenté certains bénéfices pour ses collaborateurs et a effectué la révision des contrats de main d'œuvre externe de courte et moyenne durée. Cette pratique, même si très utilisée dans l'industrie du pétrole, apporte souvent des doutes par rapport à la fragilité des relations de travail entre l'organisation qui fait l'embauchage et celui embauchée.

Afin de mieux pouvoir analyser les événements critiques de chacune des études de cas présentées, elles seront résumées selon les étapes de gestion de crise (Roux-Dufort, 2003) :

Tableau 2 - Le Navire NP, la Plateforme PP et les étapes de gestion des crises

\begin{tabular}{|c|c|c|}
\hline $\begin{array}{c}\text { Actions de l'entreprise A et } \\
\text { le navire NP }\end{array}$ & Phases de la crise & $\begin{array}{c}\text { Actions de l'entreprise B et } \\
\text { la plateforme PP }\end{array}$ \\
\hline $\begin{array}{l}\text { Réfutation de toute } \\
\text { responsabilité. } \\
\text { Silence : pas de réponse } \\
\text { immédiate à ses parties } \\
\text { prenantes ni aux médias. }\end{array}$ & $\begin{array}{l}\text { La mise en relation avec les } \\
\text { médias et l'occupation du } \\
\text { terrain médiatique }\end{array}$ & $\begin{array}{l}\text { Présence massive des médias } \\
\text { et des téléspectateurs pendant } \\
\text { tout le déroulement de la crise. } \\
\text { L'entreprise n'a pas laissé } \\
\text { d'espace aux rumeurs. }\end{array}$ \\
\hline $\begin{array}{l}\text { Activation immédiate d'une } \\
\text { cellule de crise. } \\
\text { Mise en place des mesures } \\
\text { pour contrôler la marée noire. }\end{array}$ & $\begin{array}{l}\text { L'activation de la cellule de } \\
\text { crise }\end{array}$ & $\begin{array}{r}\text { Mobilisation de plusieurs } \\
\text { bateaux et techniciens afin de } \\
\text { contrôler l'émergence sur le } \\
\text { lieu des explosions. }\end{array}$ \\
\hline $\begin{array}{l}\text { Plusieurs riverains et } \\
\text { associations ont dû prendre en } \\
\text { charge le nettoyage des côtes. }\end{array}$ & $\begin{array}{l}\text { La prise en charge des } \\
\text { victimes }\end{array}$ & $\begin{array}{l}\text { Prise en charge des victimes et } \\
\text { de leurs familles comme } \\
\text { première réponse à l'accident. }\end{array}$ \\
\hline $\begin{array}{l}\text { Reconnaissance tardive de la } \\
\text { gravité de la situation. } \\
\text { Prise de contrôle tardive de la } \\
\text { situation communicationnelle. }\end{array}$ & Le diagnostic de la crise & $\begin{array}{r}\text { Reconnaissance immédiate de } \\
\text { la gravité des événements. } \\
\text { Peu de coopération avec les } \\
\text { enquêtes. }\end{array}$ \\
\hline $\begin{array}{l}\text { Acceptation d'une } \\
\text { responsabilité morale } \\
\text { concernant le navire et sa } \\
\text { cargaison. }\end{array}$ & $\begin{array}{l}\text { Le déploiement des } \\
\text { dispositifs de réduction de la } \\
\text { crise et mise en ouvre du } \\
\text { redéploiement }\end{array}$ & $\begin{array}{r}\text { Développement de nouvelles } \\
\text { normes de sécurité. } \\
\text { Investissement en } \\
\text { entraînement et infrastructure. }\end{array}$ \\
\hline $\begin{array}{l}\text { Reconnaissance de son } \\
\text { manque de communication et } \\
\text { du fait que celle-ci n'était pas } \\
\text { adaptée à la situation. }\end{array}$ & $\begin{array}{l}\text { L'affinage des la stratégie de } \\
\text { communication de crise et la } \\
\text { réduction des rumeurs }\end{array}$ & $\begin{array}{l}\text { Projet de communication pour } \\
\text { la divulgation des enquêtes. } \\
\text { Discussion ouverte avec toutes } \\
\text { ses parties prenantes. }\end{array}$ \\
\hline $\begin{array}{l}\text { Gestion des boycottes et de } \\
\text { l'image d'entreprise (très }\end{array}$ & $\begin{array}{c}\text { La perception de } \\
\text { l'entreprise et la gestion des }\end{array}$ & $\begin{array}{r}\text { Plus de bénéfices pour les } \\
\text { collaborateurs. }\end{array}$ \\
\hline
\end{tabular}


endommagée).

Peu de conséquences au

niveau financier.

Développement d'une

politique de «pré-gestion »

des crises. effets secondaires

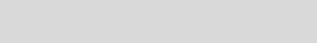

Révision des contrats de main d'œuvre externe de courte et moyenne durée.

L'entreprise a pu préserver en partie ce qu'elle symbolise devant l'opinion publique.

Source : Tableau construit selon les travaux de Roux-Dufort (2003)

\subsection{LES CARACTERISTIQUES DES CRISES DANS LES ENTREPRISES A ET B}

L'analyse des épisodes de crise selon les approches événementielles et processuelles sera exposée. Pour des raisons de clarté, les contributions des interviewés seront présentées à interligne simple, à la droite du texte.

\subsubsection{L’approche événementielle}

En analysant la crise du navire pétrolier NP affrontée par l'entreprise A selon l'approche événementielle, il faut d'abord se concentrer sur le concept d'accident que cela englobe (Lagadec, 1991). Lorsque cette approche se caractérise par un événement «brutal et ponctuel » dont l'imprévisible et l'improbable sont les caractéristiques principales (Roux-Dufort, 2000), l'élément déclencheur est clair et en apparence peu prévisible : la destruction du navire dans une mer d'une force extraordinaire. Trois des experts interviewés donnent leur perception de la crise :

\footnotetext{
«Perturbation importante du système et risque pour l'existence de l'entreprise » (Interviewé 4)

"C'est une situation que ne peut pas être contrôlée avec les procédures de routine des organisations » (Interviewé 3)

« Une crise doit être affrontée comme une opportunité » (Interviewé 2)
}

L'événement peut être considéré comme la cause de la crise, dans un premier temps. Ensuite, il faut se concentrer sur les citernes du navire NP, où il avait plusieurs milliers de tonnes de fioul lourd. Dans la logique événementielle, dont l'observation des événements est faite plutôt sous l'angle des conséquences de la crise (Roux-Dufort, 2000), il y a une marée noire de plusieurs centaines de kilomètres qui a touché une partie importante des côtes françaises. Dans le cas de l'entreprise B, et selon les mêmes critères, l'approche événementielle permet d'identifier un accident qui a déclenché toute une situation de crise. La cause: trois grandes explosions sur une plateforme pétrolière. Immédiatement, des conséquences tragiques : la mort des ouvriers. 
Ainsi, selon la logique événementielle, les crises du navire NP et de la plateforme PP peuvent être représentées par les schémas suivants :

Figure 4 - Le navire pétrolier NP, la plateforme pétrolière PP et l'approche événementielle
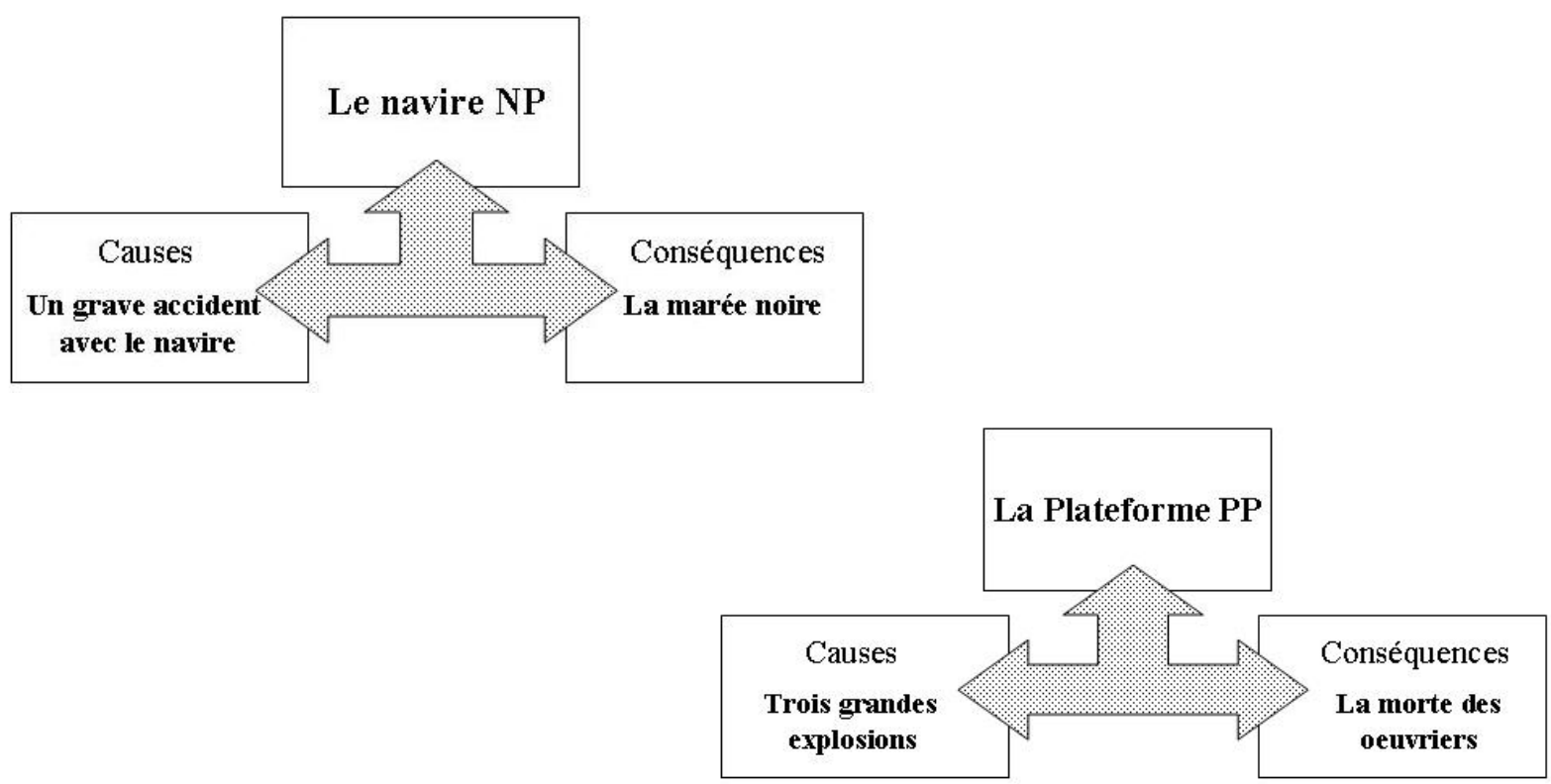

L’approche événementielle se centre sur l'événement déclencheur. Les deux cas présentés comportent des informations ponctuelles qui sont capables de donner quelques réponses pour essayer de comprendre les événements. Telle logique d'analyse appréhende la crise comme si elle avait commencé lorsque le navire s'est coupé en deux ou lorsque la première explosion a eu lieu. Il y a une tendance à confondre la crise avec son événement déclencheur (Roux-Dufort, 2000), et à essayer de la résoudre en délimitant la ligne d'action sur un événement spécifique, sans marquer d'intérêt pour ce qui se trouve « autour » de son déclencheur :

«Il faut affronter immédiatement la situation, diagnostiquer le problème, prendre soin des victimes et communiquer » (Interviewé 2)

L'approche événementielle peut se circonscrire à la stricte limitation des événements de crise de façon à ne pas laisser l'événement perturber le bon fonctionnement de l'organisation (Forgues, 1996) et, par conséquent, elle ne considère pas la situation d'une façon holistique et processuelle, dans le long et le court terme.

«Les collaborateurs, les clients, les actionnaires, les fournisseurs, la presse, la société et le gouvernement doivent être informés des problèmes et des actions menées pour les résoudre » (Interviewé 4) 
« A court terme, il faut préserver la santé des victimes et minimiser les dégâts physiques à travers le contrôle des événements. A long terme, il faut préserver les finances de l'entreprise en évitant la diffusion d'informations erronées par les media (...) mépriser la perception de l'opinion publique et concentrer les efforts seulement sur les aspects techniques de la gestion de crise, c'est une erreur » (Interviewé 3)

Il y a ainsi des défauts importants en ce qui concerne l'approche événementielle, lorsqu'on privilégie l'événement déclencheur (l'accident) et ses conséquences. Roux-Dufort (2003) souligne que cette approche «englobe une somme d'événements et de situations très variés ce qui fait perdre le pouvoir de précision et de discernement ». Puisqu'elle privilégie les effets, les gestionnaires ne peuvent se prononcer sur l'état de crise qu'à partir du moment où elle se déclenche, lorsqu'il est déjà trop tard. D'après l'auteur, attendre l'évaluation des conséquences pour se prononcer est « adopter une attitude attentiste et défensive trop dangereuse ».

D'après cette analyse, l'approche événementielle laisse des lacunes concernant les réponses exigées par les crises : quel était l'état du navire avant l'accident? Est-ce que la crise a commencé avec les explosions ? Où sont les "racines" organisationnelles des crises ? Si la marée noire et la mort des ouvriers ont été les conséquences, quelles ont été les «conséquences des conséquences »? Ce sont de questions pour lesquelles l'approche événementielle n'apporte pas de réponses en raison de son point de vue restrictif (Roux-Dufort, 2003).

Afin de répondre à ces questions d'une façon plus complète, les deux cas de crise vont à présent être analysés à la lumière de l'approche processuelle.

\subsubsection{L'approche processuelle}

Selon une logique processuelle, la crise est un processus qui, à partir d'un événement clé, déclenche «une inadéquation soudaine du cadre d'action de l'entreprise », ce qui fait que ses parties prenantes perdent leurs références (Roux-Dufort, 2003). Pour analyser une crise selon cette approche, il faut d'abord considérer quatre éléments : ses origines, ses caractéristiques, se(s) élément(s) déclencheur(s) et son développement (Roux-Dufort, 2000).

Pour l'entreprise A et le navire pétrolier NP, les questions sur les origines de cette crise sont très liées à l'origine et à l'état de conservation du navire. Cependant, ce qui complique cette question est, en fait, le problème de la culpabilité : à qui la faute ? L'entreprise A a nié, dès le début de l'affaire, toutes les charges en invoquant la responsabilité juridique, vu que le navire ne lui appartenait pas. Néanmoins, selon certains auteurs, en termes moraux l'entreprise était 
impliquée. Plusieurs questions sur ce sujet pourraient trouver une réponse à travers d'une enquête plus approfondie, ce qui n'est pas au centre de cette recherche.

En plus des «questions administratives », il faut rappeler que les conditions climatiques de la mer, le jour du naufrage du navire, n'étaient pas du tout favorables, ce qui pourrait être considéré comme l'événement déclencheur de la crise.

En ce qui concerne les caractéristiques de cette crise, l'entreprise A a dû faire face à «l'intrusion » de plusieurs nouveaux acteurs dans leur cadre référentiel (Libaert, 2005) : la forte présence des media et des personnes affectés par la marée noire, spécialement celles impliquées dans le nettoyage des côtes. Il y a eu, de plus, de grandes dépenses pour l'entreprise : plusieurs millions d'euros pour les dommages, sans compter les effets sur l'environnement. Concernant le développement de la crise du navire pétrolier NP, à l'entreprise A cela a consisté de la formation immédiate d'une cellule de crise et à la création d'une mission pour combattre la marée noire. Cependant, même si à un moment donné l'entreprise $\mathrm{A}$ a décidé de prendre le contrôle de la crise, selon plusieurs media elle a eu un retard presque impardonnable avant de commencer à agir.

\footnotetext{
«Une erreur? Ne pas communiquer : si vous avez une mauvaise nouvelle, vous devez la communiquer vite, avant que quelqu'un ne le fasse pour vous (...) Les personnes perçoivent très rapidement les choses. Nous vivons dans un reality-show permanent » (Interviewé 2).

«C'est une des premières choses lors d'une crise (...) la communication attire l'attention sur la manière dont la gestion de la crise est perçue. S'il y a un grand décalage entre l'effet et la perception, ça peut générer une crise » (Interviewé 1)
}

Le manque d'action par l'entreprise a provoqué une forte réaction de l'opinion publique, qui a répondu avec des boycotts et des protestations. Juste après l'accident, des actions judiciaires et des enquêtes ont commencé à être menées par plusieurs associations et communautés touchées par les événements. Le processus de crise du navire pétrolier NP peut être représenté par le schéma suivant :

Figure 5 - Le navire pétrolier NP et l'approche processuelle 


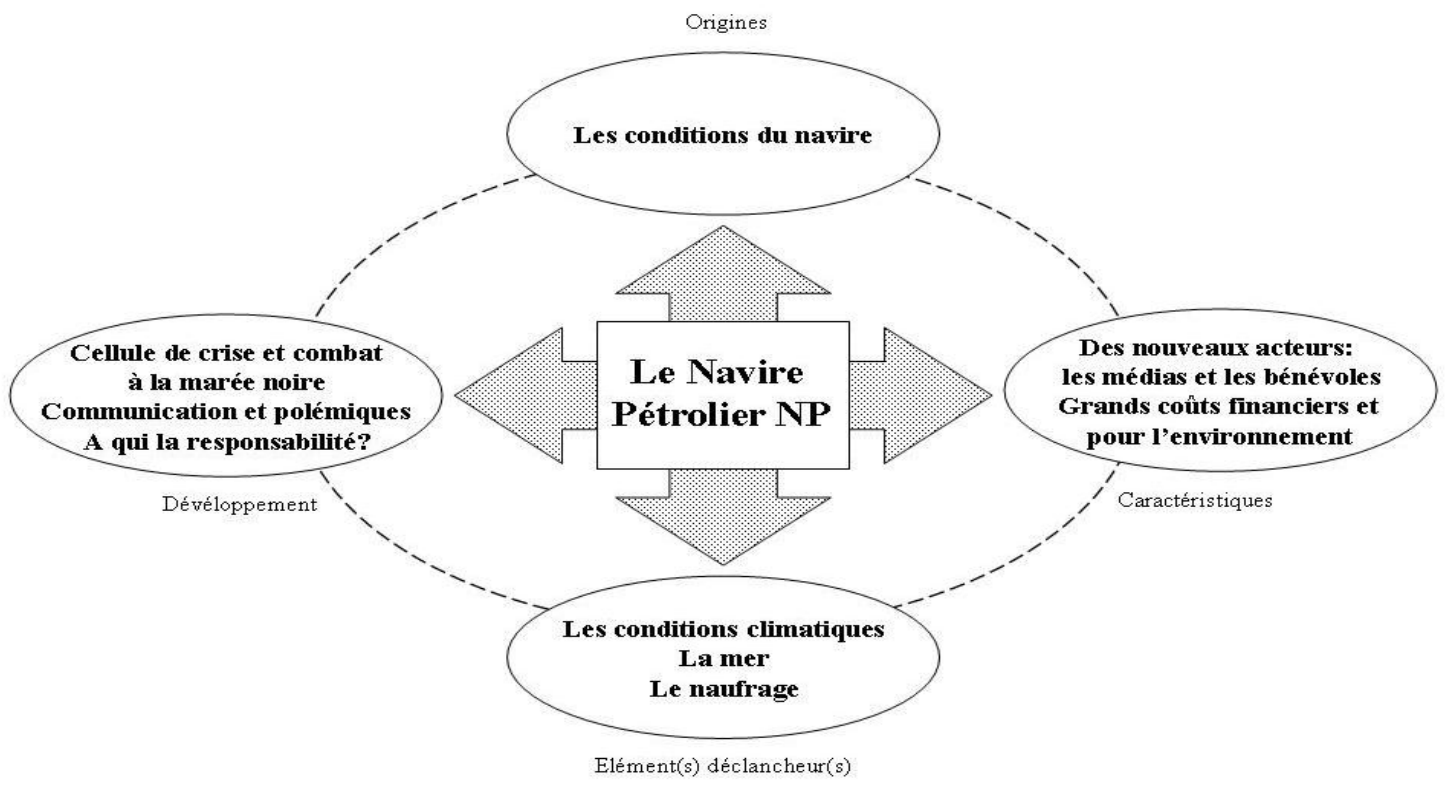

En ce qui concerne l'entreprise B, il est possible d'appréhender le cas de la plateforme PP à partir de l'approche processuelle. Telle crise n'a pas eu ses origines uniquement à cause des problèmes techniques in situ: elle était beaucoup plus enracinée dans les pratiques de l'organisation. Selon la documentation analysée, l'entreprise B n'a pas fait attention aux recommandations des organisations syndicales à l'occasion d'autres accidents. Selon la presse, l'entreprise aurait ignoré certains problèmes qui demandaient la suspension des travaux pendant quelques jours. Dans ce cas, il est possible d'identifier l'événement déclencheur de la crise par les explosions, mais identifier ses origines devient beaucoup moins évident.

La principale caractéristique de la crise de la plateforme PP a été la saturation de la capacité de communication de l'entreprise (Libaert, 2005) liée au grand intérêt des media. La plateforme est devenue un événement médiatique au Brésil, avec une grande dramatisation des faits. Tout le pays a accompagné cet épisode qui a duré cinq jours, jusqu'à ce que la dernière partie de la plateforme soit disparue sous l'eau.

«Il faut être la source officielle des informations pour les contrôler » (Interviewé 2)

« Moi, je vais vous dire qu'il y a des rôles et quelque soit la manière dont vous gérez la crise, vous êtes enfermé dans un rôle (...) c'est une caractéristique des media : vous avez les bons et vous avez les méchants » (Interviewé 1)

Le développement de cette crise a été fortement basé sur la communication : l'entreprise B a toujours parlé de façon transparente avec les media et toutes les personnes concernées par la 
tragédie. Avant de parler à la presse, la cellule de crise a contacté toutes les familles des victimes et des collaborateurs présents sur la plateforme au moment de l'accident pour donner des informations et éviter la panique (selon documentation divulguée par l'entreprise B elle même).

« C'est fondamental. A travers la communication, on arrive à avoir l'engagement et la compréhension des publics sur la résolution des problèmes » (Interviewé 5)

Le processus de crise de la plateforme PP peut être représenté par le schéma suivant :

Figure 6 - La plateforme pétrolière PP et l'approche processuelle

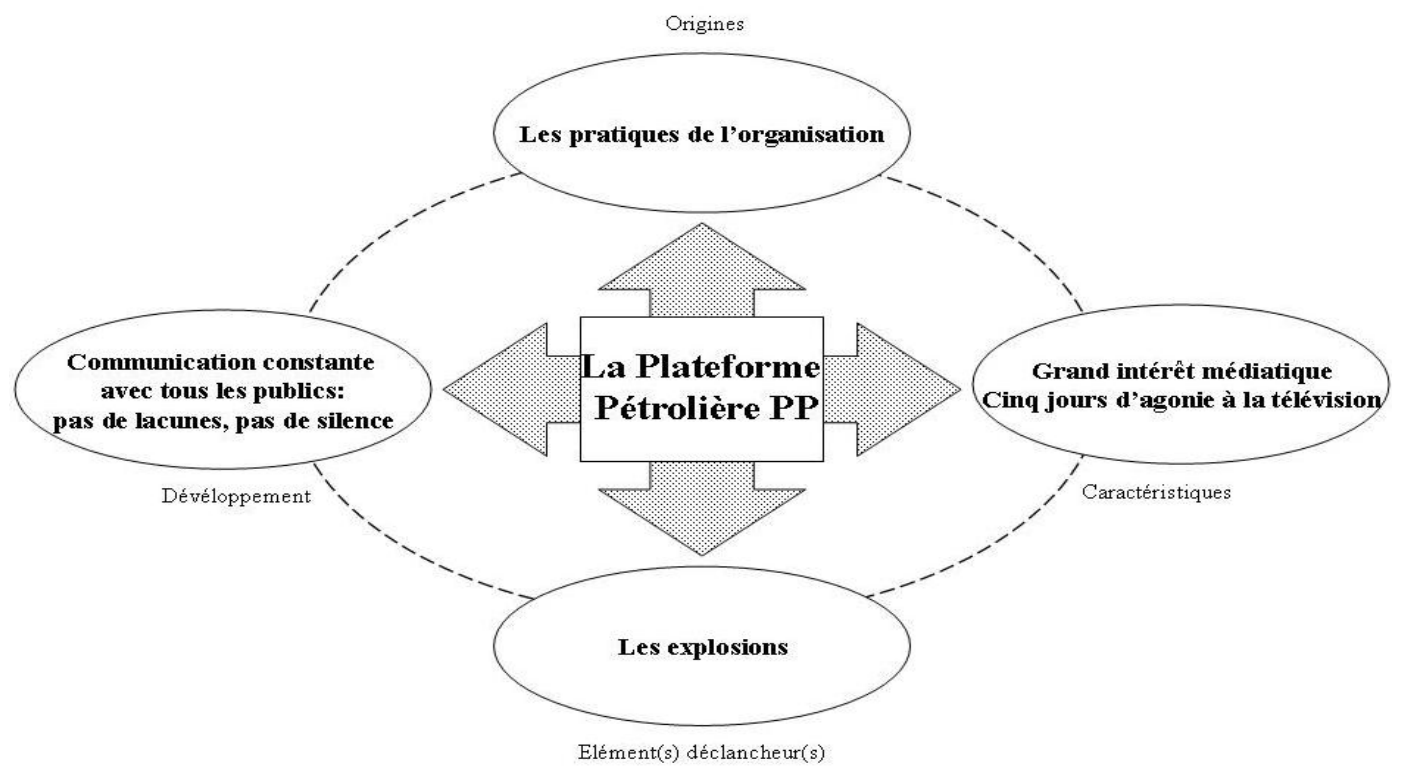

\section{DISCUSSION}

Après avoir analysé les deux exemples, il est possible observer que l'approche processuelle permet une visualisation plus complète des situations critiques par rapport à l'approche événementielle (Roux-Dufort, 2005). Puisque une crise est toujours un événement unique dans ses caractéristiques et n'a jamais une seule raison pour se produire, un « raisonnement causal et linéaire » ne permet pas aux organisations de comprendre toutes ses nuances (Roux-Dufort, 2000). La crise est beaucoup plus qu'un événement isolé, il s'agit d'un processus inhérent à l'apprentissage et à l'évolution de l'organisation.

Tableau 3 - Les entreprises A e B et les approches événementielle et processuelle 


\begin{tabular}{|c|c|c|}
\hline \multirow{2}{*}{$\begin{array}{l}\text { Dimension } \\
\text { d'analyse }\end{array}$} & Approche Evénementielle & Approche Processuelle \\
\hline & \multicolumn{2}{|c|}{ (Caractéristiques des dimensions selon chaque approche en italique) } \\
\hline $\begin{array}{l}\text { Nature de } \\
\text { l'occurrence } \\
\text { des } \\
\text { crises }\end{array}$ & $\begin{array}{l}\text { - Surprise } \\
\text { - La crise est imprévisible } \\
\text { A - Déclenchement de la crise par la } \\
\text { destruction du navire à cause d'une } \\
\text { tempête. } \\
\text { B - Crise déclenchée par trois } \\
\text { explosions sur la plateforme } \\
\text { pétrolière. }\end{array}$ & $\begin{array}{l}\text { - Etapes définies et progression de la } \\
\text { crise dans l'intensité et la visibilité } \\
\text { - La crise est précédée de signes avant- } \\
\text { coureurs } \\
\text { A - Plusieurs facteurs déclencheurs : } \\
\text { l'origine du navire, sa documentation, les } \\
\text { conditions de la mer, la responsabilité } \\
\text { pour la cargaison. } \\
\text { B - L'entreprise avait déjà eu des } \\
\text { accidents de travail dans le passé. La } \\
\text { crise était beaucoup plus enracinée dans } \\
\text { les pratiques de l'entreprise. }\end{array}$ \\
\hline Fréquence & $\begin{array}{l}\text { - La crise est improbable } \\
\text { A - L'accident avec le navire a été } \\
\text { une situation inattendue... } \\
\text { B - ... aussi bien que les explosions } \\
\text { dans la plateforme. } \\
\text { Les entreprises ne s'attendaient pas à } \\
\text { devoir affronter des telles situations. }\end{array}$ & $\begin{array}{l}\text { - Les crises sont des événements rares } \\
\text { mais normaux et inhérents aux systèmes } \\
\text { A - L'entreprise avait déjà des } \\
\text { disfonctionnements qui ont favorisé les } \\
\text { événements critiques, comme le manque } \\
\text { de soin pour choisir ses navires. } \\
\text { B - Des facteurs liés aux pratiques de } \\
\text { gestion (au niveau de l'embauche des } \\
\text { intérimaires, par exemple) ont également } \\
\text { poussé la société vers la crise. }\end{array}$ \\
\hline $\begin{array}{l}\text { Angles } \\
\text { d'observation } \\
\text { des crises }\end{array}$ & $\begin{array}{l}\text { - Les conséquences des crises } \\
\text { A - Une marée noire de plusieurs } \\
\text { centaines de kilomètres. } \\
\text { B - La mort de quelques ouvriers de la } \\
\text { compagnie. }\end{array}$ & $\begin{array}{l}\text { - Dynamique d'occurrence, } \\
\text { d'amplification et de résorption des } \\
\text { événements } \\
\text { Dans une logique processuelle, les } \\
\text { conséquences ont été beaucoup plus } \\
\text { complexes: les crises ont généré « une } \\
\text { inadéquation soudaine du cadre d'action } \\
\text { de l'entreprise », où les parties prenantes } \\
\text { n'étaient plus capables de travailler } \\
\text { ensemble. } \\
\text { A - Endommagement de l'image, } \\
\text { boycottes, mise en place de nouvelles } \\
\text { procédures de gestion de crise. } \\
\text { B - Pertes économiques, présence } \\
\text { massive des médias, révision de ses } \\
\text { pratiques d'embauche,... }\end{array}$ \\
\hline $\begin{array}{l}\text { Sources des } \\
\text { crises }\end{array}$ & $\begin{array}{l}\text {-L'événement déclencheur (centrée } \\
\text { sur le symptôme) } \\
\text { A - L'accident avec le navire. } \\
\text { B - L'explosion de la plateforme }\end{array}$ & $\begin{array}{l}\text { - L'interaction non linéaire de multiples } \\
\text { facteurs et acteurs (centrée sur la } \\
\text { dynamique d'occurrence) } \\
\text { A- Pratiques administratives mal résolues } \\
\text { au niveau du choix et de la vérification } \\
\text { des bateaux frétés. }\end{array}$ \\
\hline
\end{tabular}




\begin{tabular}{|c|c|c|}
\hline & & $\begin{array}{l}\text { B - Modèle de gestion qui donnait } \\
\text { priorité à la vitesse et aux buts de } \\
\text { production, au lieu de privilégier la } \\
\text { sécurité et l'entraînement du personnel. }\end{array}$ \\
\hline $\begin{array}{l}\text { Explication des } \\
\text { crises }\end{array}$ & $\begin{array}{l}\text { - Principe de cause à effet } \\
\text { A et B - Tendance à confondre la } \\
\text { crise avec son événement } \\
\text { déclencheur. } \\
\text { A - Mer violente = navire brisé = } \\
\text { marée noire } \\
\text { B - Explosion et morts des ouvriers }\end{array}$ & $\begin{array}{l}\text { - Approche systémique des événements de } \\
\text { crise } \\
\text { A et B - Analyse des (a) origines de la } \\
\text { crise, (b) ses caractéristiques, (c) se(s) } \\
\text { élément(s) déclencheur(s) et (d) son } \\
\text { développement. } \\
\text { A - (a) les conditions du navire; (b) des } \\
\text { nouveaux acteurs, grands coûts financiers } \\
\text { et environnementaux ; (c) les conditions } \\
\text { climatiques et de la mer, le naufrage ; (d) } \\
\text { la marée noire, les polémiques. } \\
\text { B - (a) les pratiques de l'organisation; (b) } \\
\text { l'intérêt médiatique ; (c) les explosions ; } \\
\text { (d) pas de lacunes communicationnelles. }\end{array}$ \\
\hline $\begin{array}{l}\text { Efforts de } \\
\text { gestion } \\
\text { des crises }\end{array}$ & $\begin{array}{l}\text { - Concentration sur la réaction de } \\
\text { l'entreprise face à la crise } \\
\text { A et B - Délimitation de la ligne } \\
\text { d'action sur un événement spécifique } \\
\text { A - Attention portée sur la question de } \\
\text { la responsabilité de l'entreprise dans } \\
\text { un premier moment. } \\
\text { B - Attention portée sur les victimes } \\
\text { et le contrôle des fuites d'huile. }\end{array}$ & $\begin{array}{l}\text { - Concentration sur la prévention, la } \\
\text { réaction et l'apprentissage } \\
\text { A - Formation immédiate d'une cellule } \\
\text { de crise, création des nouvelles pratiques } \\
\text { de gestion de crise, apprentissage au } \\
\text { niveau des rapports avec les médias. } \\
\text { B - Formation immédiate d'une cellule } \\
\text { de crise, mise en place des nouvelles } \\
\text { pratiques de sécurité du travail, } \\
\text { apprentissage au niveau des pratiques en } \\
\text { ressources humaines. }\end{array}$ \\
\hline $\begin{array}{l}\text { Attitude de } \\
\text { l'organisation } \\
\text { vis-à-vis des } \\
\text { crises }\end{array}$ & $\begin{array}{l}\text {-Attentisme } \\
\text { - Fatalisme } \\
\text { A - Attente de l'évaluation des } \\
\text { conséquences des événements pour se } \\
\text { prononcer sur l'état de crise. } \\
\text { B - Prise en compte des informations } \\
\text { sur le dysfonctionnement de ses } \\
\text { pratiques de manière passive. }\end{array}$ & $\begin{array}{l}\text { - Pro activité } \\
\text { A - Le manque d'action par l'entreprise a } \\
\text { provoqué une forte réaction de l'opinion } \\
\text { publique (boycotts et protestations) } \\
\text { B - Gestion de crise basée sur la } \\
\text { communication active et transparente } \\
\text { avec les personnes concernées. }\end{array}$ \\
\hline
\end{tabular}

Source : Tableau construit selon les travaux de Roux-Dufort (2000)

Comme le montre le tableau ci-dessus, chaque dimension d'un événement de crise peut être examinée à travers de deux approches : événementielle et processuelle. Cependant, d'après l'analyse des études de cas sous la lumière de la littérature mobilisée dans ce travail, l'approche processuelle s'est confirmée comme la plus adaptée, voir complète, pour appréhender les situations critiques. Dans le cas de la nature de l'occurrence des crises, les études de cas ont 
montré que les accidents n'étaient pas tout à fait des surprises, puisque d'autres éléments (comme les origines du navire dans un cas; et les accidents de travail, dans un autre) avaient antérieurement été perçus. C'étaient des «disfonctionnements cumulés et potentiellement repérables »dans le quotidien des entreprises (Thiétart et Forgues, 1997), mais qui ont été ignorés. Concernant la fréquence des crises, les entreprises ne s'attendaient pas à devoir subir des telles situations, même si elles avaient déjà affronté des épisodes similaires. Encore une fois, la crise a été considérée comme imprévisible (Bryson, 1981), même si elle concerne un processus dont les phases d'installation, d'évolution et de développement sont, dans la plupart des cas, identifiables (Turner, 1976 ; Roux-Dufort, 2003).

Concernant les angles d'observation des crises, on observe qu'ils n'étaient pas centrés sur une seule conséquence des événements critiques étudiés, mais sur plusieurs. Une situation de crise ne doit pas être analysée comme un fait ponctuel et limité dans le temps et l'espace (comme proposé par Lagadec, 1991, dans sa définition d'accident), mais plutôt comme une situation pleine de significations, d'acteurs et de victimes (Marcus et Goodman, 1991). Dans les cas étudiés, les entreprises n'ont pas pu se centrer uniquement sur la marée noire ou la mort des ouvriers : elles ont dû considérer les autres aspects touchés par les crises, comme leurs parties prenantes et leur contexte socioculturel, par exemple. Dans le cas des sources des crises, plusieurs facteurs doivent être examinés pour les analyser, et non seulement les événements déclencheurs. Sous cet aspect, il faut «embrasser la crise dans un laps de temps et un espace élargi » (Forgues, 1996), c'est-à-dire, prend en compte les pratiques administratives mal résolues ou les modèles de gestion mal formulés pour trouver les origines des événements critiques. Ceci consiste à situer la crise dans un contexte plus riche qui concerne ses origines, son incubation et sa «dynamique de développement » (Turner, 1976 ; Roux-Dufort, 2000).

Essayer de trouver une explication des crises dans les cas analysés peut être assez difficile, justement à cause du grand nombre d'aspects à être considérés. Comment expliquer l'accident concernant l'entreprise A uniquement en utilisant la mer violente comme principale raison, ou celui de l'entreprise B en disant que la faute était des explosions ? Une telle analyse demande une approche systémique de la situation, puisque ce sont des aspects inévitables qui font déjà partie du système de l'entreprise (Perrow, 1984 ; Forgues, 1996) et qui ne peuvent pas être ignorés. Pendant que les dirigeants de l'entreprise A concentraient leurs efforts de gestion des crises en nier leur responsabilité ; ou que l'entreprise B ne voulait pas admettre que leur 
modèle de gestion avait des problèmes, les deux cas de crise ne montraient pas signes de résolution. Par leur caractère multiforme, les crises concernent plusieurs domaines (Libaert, 2005) et la simple réaction aux événements ne serait pas suffisante pour résoudre les problèmes. Dans une approche plus complète, il ne faut pas uniquement réagir aux événements critiques, mais voir la crise comme une opportunité en passant par une phase de rééquilibrage, où l'entreprise peut opter soit pour retourner au statu quo, soit initier un changement profond (RouxDufort, 2003). Cette dernière notion d'opportunité amène cette analyse à la question de l'attitude de l'organisation vis-à-vis des crises. Les entreprises A et B ont toutes les deux présenté des attitudes attentiste et fataliste concernant leurs crises, soit en laissant des lacunes médiatiques, soit en ignorant les dysfonctionnements au sein de ses pratiques d'embauche, respectivement. Dans une vision plus optimiste de ces événements, en prenant la crise comme une opportunité avec un fort pouvoir d'évolution, un « déclencheur» de changements ou même un processus de transformation (Bryson, 1981 ; Shrivastava et all., 1988 ; Shrivastava, 1993 ; Ogrizek et Guillery, 1997 ; Roux-Dufort, 2000 ; Libaert, 2005), les entreprises auraient pu éviter certains situations, comme les boycottes, dans le cas de l'entreprise A, par exemple.

\section{CONCLUSION}

Dans ce travail de recherche, nous avons élaboré deux études de cas de crise et analysé ses différentes dimensions sous la lumière des approches événementielle et processuelle. Ceci nous a permis d'arriver à quelques constatations concernant ces deux modèles théoriques, qui ne doivent cependant pas être interprétées comme des conclusions définitives, mais des résultats d'une des plusieurs étapes qui doivent encore être consacrées aux études de la gestion des crises.

Ce travail apporte quelques contributions pour l'étude des crises dans la mesure qu'il analyse et met en discussion les différentes façons de regarder les situations critiques, même si dans un même secteur d'activité, comme est le cas des entreprises choisies pour cet article. Dans cette démarche, l'entretien en profondeur est ressorti comme un " filtre » intéressant pour la gestion des crises, qui a permis l'analyse détaillée de certains de ses aspects pratiques au sein des organisations, ce qui peut être observé à travers des verbatim ajoutés au texte.

Du point de vu managériale, l'analyse des dimensions de crise nous a permis de confirmer l'approche processuelle comme celle capable de mieux cerner cette question. Nous avons également pu mettre en évidence quelques points qui pourraient être utiles aux membres des 
cellules de crise. Une approche systémique de la crise et de ses origines, la capitalisation sur les expériences vécues par l'organisation et une posture optimiste et proactive des gestionnaires par rapport aux événements critiques ressortent de cette étude comme éléments clé de la gestion des crises et de ses conséquences dans le contexte et avec les parties prenantes de l'entreprise.

Du point de vue de la société, autant que du public des crises, ce travail a pu apporter des informations sur le fonctionnement des tels événements et ainsi permettre aux différentes parties prenantes de mieux comprendre ses enjeux. En ce qui concerne les organisations, il a pu également apporter quelques exemples de gestion des crises et, dans le sens pratique de la gestion, peut-être même attirer l'attention des cadres sur la réflexion nécessaire sur les méthodes et sur l'importance de la formation pour la gestion de crise.

Ce travail peut également être marqué par certaines limites, comme l'impossibilité d'avoir accès à certaines documentations, spécialement sur les cas de crise des entreprises. Concernant les entretiens, il y a eu quelques difficultés pour trouver des personnes intéressées à nous parler : le sujet «crise » et le retour sur certaines expériences critiques ne sont pas faciles de faire ressortir, ce qui a limité le nombre d'entretiens réalisés et ce qui nous considérons comme une limite significative de notre travail. Il serait également souhaitable de réaliser des études de cas dans d'autres domaines d'activité, pour lesquelles les conséquences des crises revêtiraient un caractère moins transparent.

Nous encourageons enfin la poursuite des études sur la crise concernant ces approches processuelles et événementielles au sein des entreprises dans le but d'approfondir les observations in situ. Cela nécessiterait toutefois d'appréhender les crises dans leur dimension génératrice et organisationnelle. Morin $(1977,1980,1986)$ propose à ce titre un processus complexe (circuit tétralogique) formant un mouvement dynamique, où les constantes interactions et rencontres entre les parties produisent des désordres, qui se développent jusqu'à arriver à un nouvel ordre qui, à son tour, participera à l'organisation du tout. Les émergences, considérées par Morin (1980) comme l'effet synergique des relations entre les parties, rendraient possible le développement de nouvelles caractéristiques que la simple addition des parties ne peut produire. C'est donc l'analyse de ces caractéristiques formées par l'interaction des parties qui pourraient permettre une étude organisationnelle approfondie et ainsi provoquer le dépassement nécessaire de l'opposition entre les approches processuelles et événementielles. 


\section{BIBLIOGRAPHIE}

Allison, G. \& P. Zelikow (1999), Essence of decision: Explaining the Cuban missile crisis $-2^{\text {nd }}$ ed., New York: Longman.

Baumard, P. \& J. Ibert (2003), Quelles approches avec quelles données ?, in R. A. Thiétart \& coll., Méthodes de recherche en management, Paris : Dunod, 82-103.

Bland, M. (1998), Communicating out of a crisis, Londres: Macmillan Business.

Bowonder, B. \& H. Linstone (1987), Notes on the Bhopal accident: Risk analysis and multiple perspectives, Technological Forecasting and Social Change, vol. 32, 183 - 202.

Bryson, J. M. (1981), A Perspective on Planning and Crises in the Public Sector, Strategic Management Journal, vol. 2, p. 181 - 196.

Daigne. J. F. (1991), Management en période de crise. Aspects stratégiques, financiers et sociaux. Paris: Les Éditions D’Organisation.

Denzin, N. K. \& I. S. Lincoln (2000), The discipline and practice of qualitative research, in N. K.

Denzin \& I. S. Lincoln, Handbook of qualitative research, Thousand Oaks (CA): Sage Publications, Introduction.

Dutton, J. E. (1986), The processing of crisis and non-crisis strategic issues, Journal of Management Studies, vol. 23, p. 501 - 517.

Eisenhardt, K. M. (1989), Building theory from case study research, Academy of Management Review, vol. 14, no. 4, $532-550$.

Forgues, B. (1996), Nouvelles approches de la gestion de crise, Revue Française de Gestion, ${ }^{\circ}$ 108, p. $72-78$.

Forgues, B. \& I. Vandangeon-Derumez (2007), Analyses Longitudinales, in R. A. Thiétart \& coll., Méthodes de recherche en management, Paris : Dunod, 422 - 448.

Freitas, C. M. (2001), Acidentes de trabalho em plataformas de petróleo, Caderno Saúde Pública, $\mathrm{n}^{\circ} 17$, p. $117-130$.

Marcus, A. \& R. Goodman (1991), Victims and shareholders: The dilemmas of presenting corporate policy during a crisis, Academy of Management Journal, vol. 34, n ${ }^{\circ}$ 2, p. 281 - 305.

Hermann, C. F. (1963), Some consequences of crisis which limit the viability of organizations, Administrative Science Quarterly, $\mathrm{n}^{\circ}$ 8, p. $61-68$.

Lagadec, P. (1991), La gestion des crises, Paris: McGraw Hill.

Lagadec, P. (1993), Preventing chaos in a crisis, London: McGraw Hill. 
Lagadec, P. (1995), Cellules de Crise: Les conditions d'une conduite efficace, Paris: Editions d'organisation.

Lagadec, P. (2000), Ruptures créatrices, Paris: Editions d'organisation.

Laubier, L. (2005), Diversidade da maré negra, Scientific American Brasil, ed. 39.

Lehu, J. M. (1998), Alerte Produit!, Paris: Editions d'organisation.

Libaert, T. (2005), La communication de crise, Paris : Dunod.

Mitroff, I. ; P. Shrivastava ; F. E. Udwadia (1987), Effective Crisis Management, The Academy of Management Executive, vol. 1, no. 3, p. 283 - 292.

Morin, E. (1976), Pour une crisologie, Communications, n 25, p. 149 - 163.

Morin, E. (1977), La méthode: la nature de la nature, Paris: Seuil.

Morin, E. (1980), La méthode: la vie de la vie, Paris: Seuil.

Morin, E. (1986), La méthode: la connaissance de la connaissance, Paris: Seuil.

Morin, E. (1994), Sociologie, Paris: Seuil.

Ogrizek, M. \& J. M. Guillery (1997), La communication de crise, Paris: Presses Universitaires de France.

Pauchant, T. \& R. Douville (1994), Recente research in crisis management: a study of 24 authors' publications from 1986 to 1991, Industrial and Environmental Crisis Quarterly, n 7, p. $43-61$.

Paz, S. P. (2002), Mareas negras, un triste costume nas costas europeas, Europe \& Liberté Magazine, ed. août.

Paschoal, G. (2002), O petróleo e a agressão ao meio ambiente, disponible le 15/11/2007 en http://www.comciencia.br/reportagens/petroleo/pet09.shtml.

Pearson, C. \& J. Clair (1998), Reframing crisis management, Academy of Management Review, vol. $23, \mathrm{n}^{\circ} 1$, p. $56-76$.

Perrow, C. (1984), Normal accidents: Living with high-risk technologies, New York: Basic Books.

Perrow, C. (1994), The limits of safety: the enhancements of a Theory of Accidents, Journal of Contingencies and Crisis Management, vol. 2, no. 4, p. 212 - 220.

Richardson, R. J. (1999), Pesquisa social: métodos e técnicas, São Paulo: Atlas.

Roux-Dufort, C. (2000), La gestion de crise : Un enjeu stratégique pour les organisations, Paris: De Boeck \& Larcier. 
Roux-Dufort, C. (2003), Gérer et décider en situation de crise, Paris: Dunod.

Roux-Dufort, C. (2005), Comment en est-on arrive là? Du terrain de crise à la catastrophe, Revue Spaces, n. 85 , p. $24-39$.

Shrivastava, P. (1993), Crisis theory/practice: Towards a sustainable future, Industrial and Environmental Crisis Quarterly, vol. 7, p. $23-42$.

Shrivastava, P. ; I. Mitroff ; D. Miller; A. Migliani (1988), Understanding Industrial Crises, Journal of Management Studies; vol. 25, no. 4, p. 285 - 303.

Shrivastava, P. \& I. Mitroff (1987), Strategic Management of Corporate Crises, Columbia Journal of World Business, vol. 22, issue 1, p. 5 - 11.

Siggelkow, N. (2007), Persuasion with case studies, Academy of Management Journal, vol. 50, no. 1 , p. $20-24$.

Thiétart, R. A. \& B. Forgues (1997), Action, structure and chaos, Organization Studies, vol. 18, $\mathrm{n}^{\circ} 1$, p. $119-143$.

Tran Thanh Tam, E. \& T. L. Peslouan (2004), Manager les situations difficiles, Paris: Editions d'organisation.

Turner, B. A. (1976), The organizational and interorganizational development of disasters, Administrative Science Quarterly, vol. 21, p. 378- 397.

Westphalen, M. H. (1992), Le Dicom, Paris: Triangle Edition.

Westphalen, M. H. (1998), Communicator - Le guide de la communication d'entreprise, Paris: Dunod.

Yin, R. K. (2003), Case study research: design and methods $-3^{\text {rd }}$ ed., London: Sage. 